\title{
Abel-Goncharov Type Multiquadric Quasi-Interpolation Operators with Higher Approximation Order
}

\author{
Ruifeng Wu $\mathbb{i D}^{1,2}$ \\ ${ }^{1}$ School of Applied Mathematics, Jilin University of Finance and Economics, Changchun 130117, China \\ ${ }^{2}$ Key Laboratory of Symbolic Computation and Knowledge Engineering of Ministry of Education, Jilin University, \\ Changchun 130012, China
}

Correspondence should be addressed to Ruifeng Wu; wuruifeng@jlufe.edu.cn

Received 29 September 2020; Revised 31 May 2021; Accepted 12 June 2021; Published 5 July 2021

Academic Editor: Beny Neta

Copyright (c) 2021 Ruifeng Wu. This is an open access article distributed under the Creative Commons Attribution License, which permits unrestricted use, distribution, and reproduction in any medium, provided the original work is properly cited.

\begin{abstract}
A kind of Abel-Goncharov type operators is surveyed. The presented method is studied by combining the known multiquadric quasi-interpolant with univariate Abel-Goncharov interpolation polynomials. The construction of new quasi-interpolants $\mathscr{L}_{m}^{\mathrm{AG}} f$ has the property of $m(m \in \mathbb{Z}, m>0)$ degree polynomial reproducing and converges up to a rate of $m+1$. In this study, some error bounds and convergence rates of the combined operators are studied. Error estimates indicate that our operators could provide the desired precision by choosing the suitable shape-preserving parameter $c$ and a nonnegative integer $m$. Several numerical comparisons are carried out to verify a higher degree of accuracy based on the obtained scheme. Furthermore, the advantage of our method is that the associated algorithm is very simple and easy to implement.
\end{abstract}

\section{Introduction}

Assume that $f$ is a function defined on a domain $[a, b] \subset \mathbb{R}$ containing $X$ and $x_{i} \in X, i=0, \ldots, N$ is some distinct points, where

$$
a=x_{0}<\cdots<x_{N}=b
$$

has the form

$$
\mathscr{L}[f ; a, b](x)=\sum_{j=0}^{N} \lambda_{j} \mathscr{X}\left(x-x_{j}\right),
$$

s.t.

$$
\mathscr{L}[f ; a, b]\left(x_{j}\right)=f\left(x_{j}\right), \quad \text { for } j=0,1, \ldots, N,
$$

where $\mathscr{X}(\cdot)$ is an interpolation kernel. Radial basis functions have been employed to solve the above interpolation problems (2) and (3) by many research fellows. Multiquadrics first introduced by Hardy [1],

$$
\phi_{j}(x)=\phi\left(x-x_{j}\right)=\sqrt{\left(x-x_{j}\right)^{2}+c^{2}}, \quad j=0,1, \ldots, N,
$$

are particularly interesting, on account of their particular convergence property $[2,3]$. In this study, we denote the multiquadrics and their shape-preserving parameter in (4) by notations $\phi_{j}(\cdot)$ and $c$, respectively. By means of accuracy, efficiency, and easy implementation, Franke [4] investigated that multiquadric interpolation is considered to be one of the most schemes in 29 interpolation methods. Based on distinct nodes $\left\{x_{j}\right\}_{j=0}^{N}$, Micchelli [5] proved that multiquadric interpolation is always solvable, but the resulting matrix in interpolation problems (2) and (3) quickly becomes illconditioned with the increase of the nodes. The well-known quasi-interpolation as a weaker form of (3) reproduces all polynomials of degree $\leq m$, that is,

$$
\mathscr{L}[p ; a, b](x)=p(x), \quad p \in \mathbb{P}_{m}, x \in \mathbb{R},
$$


where $\mathbb{P}_{m}=\{p$ : $\operatorname{deg}(p) \leq m\}$. In this study, we will apply the quasi-interpolation scheme to overcome the ill-conditioning problem.

Beatson and Powell [6] first constructed a univariate quasi-interpolant $\mathscr{L}_{B}$ which reproduces constants. Wu and Schaback [7] introduced another quasi-interpolant $\mathscr{L}_{D}$ possessing shape-preserving and linear-reproducing properties. They proved that the error of the operator $\mathscr{L}_{D}$ is $\mathcal{O}\left(h^{2}|\ln h|\right)$ when the shape parameter $c=\mathcal{O}(h)$ and $h=\max _{1 \leq j \leq n}\left\{\left|x_{j}-x_{j-1}\right|\right\}$. Based on the operator $\mathscr{L}_{D}$ in [7], Ling [8] provided a multilevel quasi-interpolant and showed that its convergence rate is $\mathscr{O}\left(h^{2.5}|\ln h|\right)$ as $c=\mathcal{O}(h)$. To increase the degree of the multiquadric quasi-interpolation operator, Feng and Zhou [9] provided a kind of multiquadric quasi-interpolants, and the operators could have any degree of exactness. At the same time, by applying the operator $\mathscr{L}_{B}$ with Hermite interpolation polynomials, Wang et al. [10] proposed a kind of improved quasi-interpolation operators $\mathscr{L}_{H_{2 m-1}}$ and gave the desired orders of convergence. By combining the operator $\mathscr{L}_{B}$ with Lidstone interpolating polynomials [11-13], Wu et al. [14] proposed a kind of Lidstone-type multiquadric quasi-interpolants $\widetilde{\mathscr{L}}_{\Lambda_{n}}$ possessing any degree of polynomial reproducibility. The authors have given that the approximating capacity of the operator $\widetilde{\mathscr{L}}_{\Lambda_{n}}$ is comparable with that of the operator $\mathscr{L}_{H_{2 m-1}}$. Furthermore, many researchers applied multiquadric quasiinterpolants to solve differential equations [15-26]. Meanwhile, Ali et al. [27] constructed the SDI using Timmer triangular patches, which are used to visualize the energy data, i.e., spatial interpolation in visualizing rainfall data.

By the means of construction idea in [10], we provide a kind of Abel-Goncharov type multiquadric quasi-interpolants by combining the operator $\mathscr{L}_{B}$ with Abel-Goncharov interpolating polynomials. The presented operators could reproduce polynomials of higher degree than $\mathscr{L}_{B}$. Under the suitable assumption of shape-preserving parameter $c$, we obtain the convergence rates of higher order. Therefore, we could derive the desired precision of the our operators with an optimal value of $c$.

The remaining organization of this study is arranged as follows. In Section 2, we give the definition of univariate Abel-Goncharov interpolation polynomials and derive three useful theorems for the error of approximation. Section 3 is devoted to construct Abel-Goncharov type multiquadric quasi-interpolants and study their approximation orders. In Section 4, numerical experiments are shown to compare the approximation capacity of our operators with that of Wang et al.'s quasi-interpolants. Finally, conclusion is given in Section 5.

\section{Univariate Abel-Goncharov Interpolation Polynomial}

We recall first Abel-Goncharov interpolation problem from [28-30]. Consider $\quad m \in \mathbb{N}, \quad a_{0}, a_{1}, \ldots, a_{m} \in \mathbb{R}$, $a_{0}<a_{1}<\cdots<a_{m-1}<a_{m}$, and suppose $f:\left[a_{0}, a_{m}\right] \longrightarrow \mathbb{R}$ is a function with the first $m$ derivatives $f^{(i)}, 0 \leq i \leq m$. For the nodes $a_{i} \in\left[a_{0}, a_{m}\right], 0 \leq i \leq m$, and the values $f^{(i)}\left(x_{i}\right)$, $0 \leq i \leq m$, we introduce the Abel-Goncharov interpolation problem of existing polynomial $P_{m}\left[f ; a_{0}, a_{1}, \ldots, a_{m}\right](x)$ of degree $m$, s.t.

$$
\left(P_{m}\left[f ; a_{0}, a_{1}, \ldots, a_{m}\right]\right)^{(i)}\left(a_{i}\right)=f^{(i)}\left(a_{i}\right), \quad 0 \leq i \leq m .
$$

The determinant of the linear system,

$$
D=\left|\begin{array}{ccccc}
1 & a_{0} & a_{0}^{2} & \cdots & a_{0}^{m} \\
0 & 1 ! & 2 a_{1} & \cdots & m a_{1}^{m-1} \\
0 & 0 & 2 ! & \cdots & m(m-1) a_{2}^{m-2} \\
\vdots & \vdots & \vdots & & \vdots \\
0 & 0 & 0 & \cdots & m !
\end{array}\right|,
$$

is always nonzero, and problem (6) has a unique solution. The Abel-Goncharov interpolation polynomial $P_{m}\left[f ; a_{0}, a_{1}, \ldots, a_{m}\right](x)$ could be expressed as follows:

$$
P_{m}\left[f ; a_{0}, a_{1}, \ldots, a_{m}\right](x)=\sum_{k=0}^{m} g_{k}(x) f^{(k)}\left(a_{k}\right),
$$

where $g_{k}(x), k=0,1, \ldots, m$ are known as the Goncharov polynomials of degree $k$ [31] with

$$
\left\{\begin{array}{l}
g_{k}^{(s)}\left(a_{s}\right)=0, \quad \text { if } k \neq s, \\
g_{k}^{(k)}(x)=1
\end{array}\right.
$$

By means of [29-31], we have

$$
\begin{aligned}
g_{0}(x) & =1, \\
g_{1}(x) & =x-a_{0}, \\
g_{k}(x) & =\int_{a_{0}}^{x} \mathrm{~d} t_{1} \int_{a_{1}}^{t_{1}} \mathrm{~d} t_{2} \ldots \int_{a_{k-1}}^{t_{k-1}} \mathrm{~d} t_{k} \\
& =\frac{1}{k !}\left[x^{k}-\sum_{j=0}^{k-1} g_{j}(x)\left(\begin{array}{c}
k \\
j
\end{array}\right) a_{j}^{k-j}\right], \quad k=2, \ldots, m .
\end{aligned}
$$

The Abel-Goncharov interpolation polynomial $P_{m}[f$; $\left.a_{0}, a_{1}, \ldots, a_{m}\right](x)$ has the following properties:

$$
\lim _{a_{m} \longrightarrow a_{0}} P_{m}\left[f ; a_{0}, a_{1}, \ldots, a_{m}\right](x)=\sum_{k=0}^{m} \frac{\left(x-a_{0}\right)^{k}}{k !} f^{(k)}\left(a_{0}\right) .
$$

Remark 1. As the nodes $a_{0}=a_{1}=\cdots=a_{m}$, the Abel-Goncharov interpolation polynomial $P_{m}\left[f ; a_{0}, a_{1}\right.$ $\left., \ldots, a_{m}\right](x)$ is the $m^{\text {th }}$ Taylor polynomial of $f$ about $a_{0}$.

The Abel-Goncharov interpolation polynomial $P_{m}\left[f ; a_{0}, a_{1}, \ldots, a_{m}\right](x)$ has the polynomial reproduction property as follows. 
Theorem 1. The Abel-Goncharov interpolation polynomial $P_{m}\left[f ; a_{0}, a_{1}, \ldots, a_{m}\right](x)$ reproduces all polynomials of degree no more $m$.

Proof. Let us verify that $P_{m}\left[f ; a_{0}, a_{1}, \ldots, a_{m}\right](x)=f(x)$, for $f(x)=x^{i}, i=0,1, \ldots, m, x \in\left[a_{0}, a_{m}\right]$. The Abel-Goncharov interpolation polynomial $P_{m}\left[f ; a_{0}, a_{1}, \ldots, a_{m}\right](x)$ can reproduce all polynomials of degree no more $m$. It is easily given that

$$
P_{m}\left[x^{i} ; a_{0}, a_{1}, \ldots, a_{m}\right](x)=x^{i}, \quad i=0,1, \ldots, m,
$$

while

$$
\begin{aligned}
& P_{m}\left[x^{m+1} ; a_{0}, a_{1}, \ldots, a_{m}\right](x) \\
& =g_{0}(x) a_{0}^{m+1}+g_{1}(x)(m+1) a_{1}^{m}+\cdots \\
& \quad+g_{m}(x)(m+1) m, \ldots, 2 \cdot a_{m} \neq x^{m+1} .
\end{aligned}
$$

For function $f \in C^{m}\left[a_{0}, a_{m}\right]$, the Abel-Goncharov interpolation formula is obtained as follows:

$$
f(x)=P_{m}\left[f ; a_{0}, a_{1}, \ldots, a_{m}\right](x)+R_{m}\left[f ; a_{0}, a_{1}, \ldots, a_{m}\right](x) .
$$

For bounds of the above remainder $R_{m}\left[f ; a_{0}, a_{1}, \ldots, a_{m}\right]$ even in points outside the interval $[c, d]$, we use the operator

$$
f \longrightarrow P_{m}\left[f ; a_{0}, a_{1}, \ldots, a_{m}\right]
$$

as acting on the space $C^{m}[c, d]$, where $c<a_{0}, a_{m}<d$. Based on Peano's kernel theorem [32], we provide the following integral expression for the remainder (14).

Theorem 2. Given $f \in C^{m}[c, d]$ and $x \in[c, d]$, for the remainder

$$
R_{m}\left[f ; a_{0}, a_{1}, \ldots, a_{m}\right](x)=f(x)-P_{m}\left[f ; a_{0}, a_{1}, \ldots, a_{m}\right](x),
$$

we consider the following integral representations:

$$
\begin{aligned}
& R_{m}\left[f ; a_{0}, a_{1}, \ldots, a_{m}\right](x) \\
& = \begin{cases}\frac{1}{(m-1) !} \int_{x}^{a_{m-1}} f^{(m)}(t) K_{a_{0}, a_{1}, \ldots, a_{m}}(x, t) \mathrm{d} t, & c \leq x \leq a_{0}, \\
\frac{1}{(m-1) !} \int_{a_{0}}^{a_{m-1}} f^{(m)}(t) K_{a_{0}, a_{1}, \ldots, a_{m}}(x, t) \mathrm{d} t, & a_{0} \leq x \leq a_{m-1}, \\
\frac{1}{(m-1) !} \int_{a_{0}}^{x} f^{(m)}(t) K_{a_{0}, a_{1}, \ldots, a_{m}}(x, t) \mathrm{d} t, & a_{m-1} \leq x \leq d,\end{cases}
\end{aligned}
$$

where

$$
K_{a_{0}, a_{1}, \ldots, a_{m}}(x, t)=(x-t)_{+}^{m-1}-\sum_{k=0}^{m-1} \frac{(m-1) !}{(m-k-1) !} g_{k}(x)\left(a_{k}-t\right)_{+}^{m-k-1},
$$

and $(\cdot)_{+}^{k}$ denotes the positive part of the $k^{\text {th }}$ power of the argument, such that

$$
(s)_{+}^{k}= \begin{cases}s^{k}, & s>0 \\ 0, & s \leq 0 .\end{cases}
$$

Proof. First say that in the interpolation polynomial (8), there are evaluations of derivatives of function $f$ up to the order $m-1$ in the interval [c,d]. Finally, the approximant (8) has the degree of exactness $m$. By using Peano's kernel theorem, we then obtain

$R_{m}\left[f ; a_{0}, a_{1}, \ldots, a_{m}\right](x)=\frac{1}{(m-1) !} \int_{c}^{d} f^{(m)}(t) K_{a_{0}, a_{1}, \ldots, a_{m}}(x, t) \mathrm{d} t$,

where (18) is provided by using the linear functional $f \longrightarrow R_{m}\left[f ; a_{0}, a_{1}, \ldots, a_{m}\right]$ to $(x-t)_{+}^{m-1}$ viewed as a function of $x$.

If $c \leq x \leq a_{0}$, then

$$
\begin{aligned}
R_{m} & {\left[f ; a_{0}, a_{1}, \ldots, a_{m}\right](x) } \\
= & \frac{1}{(m-1) !} \int_{c}^{x} f^{(m)}(t) K_{a_{0}, a_{1}, \ldots, a_{m}}(x, t) \mathrm{d} t \\
& +\frac{1}{(m-1) !} \int_{x}^{a_{0}} f^{(m)}(t) K_{a_{0}, a_{1}, \ldots, a_{m}}(x, t) \mathrm{d} t \\
& +\frac{1}{(m-1) !} \int_{a_{0}}^{a_{1}} f^{(m)}(t) K_{a_{0}, a_{1}, \ldots, a_{m}}(x, t) \mathrm{d} t \\
& +\frac{1}{(m-1) !} \int_{a_{1}}^{a_{2}} f^{(m)}(t) K_{a_{0}, a_{1}, \ldots, a_{m}}(x, t) \mathrm{d} t \\
& +\cdots+\frac{1}{(m-1) !} \int_{a_{m-2}}^{a_{m-1}} f^{(m)}(t) K_{a_{0}, a_{1}, \ldots, a_{m}}(x, t) \mathrm{d} t \\
& +\frac{1}{(m-1) !} \int_{a_{m-1}}^{d} f^{(m)}(t) K_{a_{0}, a_{1}, \ldots, a_{m}}(x, t) \mathrm{d} t .
\end{aligned}
$$

Let $t \in[c, x]$, then

$$
\begin{aligned}
K_{a_{0}, a_{1}, \ldots, a_{m}}(x, t) & =(x-t)^{m-1}-\sum_{k=0}^{m-1} \frac{(m-1) !}{(m-k-1) !} g_{k}(x)\left(a_{k}-t\right)^{m-k-1} \\
& =(x-t)^{m-1}-(x-t)^{m-1}=0,
\end{aligned}
$$

where $(x-t)^{m-1}$ is expressed as a polynomial in $x$ of degree $m-1$.

Let $t \in\left[a_{m-1}, d\right]$, then 


$$
K_{a_{0}, a_{1}, \ldots, a_{m}}(x, t)=0 .
$$

Thus, the first case of (17) is proved by the above process. The rest of the expressions may be obtained by an analogous manner.
The following theorem provides the desired bounds.

Theorem 3. Given $f \in C^{m}[c, d]$ and $x \in[c, d]$, for the remainder (16), we have

$$
\left|R_{m}\left[f ; a_{0}, a_{1}, \ldots, a_{m}\right](x)\right| \leq \begin{cases}C(m)\left\|f^{(m)}\right\|_{\infty}\left(a_{m-1}-x\right)^{m}, & c \leq x \leq a_{0}, \\ C(m)\left\|f^{(m)}\right\|_{\infty}\left(a_{m-1}-a_{0}\right)^{m}, & a_{0} \leq x \leq a_{m-1}, \\ C(m)\left\|f^{(m)}\right\|_{\infty}\left(x-a_{0}\right)^{m}, & a_{m-1} \leq x \leq d,\end{cases}
$$

where $\|\cdot\|_{\infty}$ denotes the sup-norm on $[c, d]$, and

$$
C(m)=1+\left(\sum_{k=0}^{m-1}+\sum_{k=1}^{m-1}+\cdots+\sum_{k=m-2}^{m-1}\right) \frac{1}{(m-k) !} .
$$

Proof. If $c \leq x \leq a_{0}$, then we have from (17),

$$
\begin{aligned}
R_{m} & {\left[f ; a_{0}, a_{1}, \ldots, a_{m}\right](x) } \\
= & \frac{1}{(m-1) !} \int_{x}^{a_{0}} f^{(m)}(t) K_{a_{0}, a_{1}, \ldots, a_{m}}(x, t) \mathrm{d} t \\
& +\frac{1}{(m-1) !} \int_{a_{0}}^{a_{1}} f^{(m)}(t) K_{a_{0}, a_{1}, \ldots, a_{m}}(x, t) \mathrm{d} t \\
& +\frac{1}{(m-1) !} \int_{a_{1}}^{a_{2}} f^{(m)}(t) K_{a_{0}, a_{1}, \ldots, a_{m}}(x, t) \mathrm{d} t \\
& +\cdots+\frac{1}{(m-1) !} \int_{a_{m-2}}^{a_{m-1}} f^{(m)}(t) K_{a_{0}, a_{1}, \ldots, a_{m}}(x, t) \mathrm{d} t .
\end{aligned}
$$

If $t \in\left[x, a_{0}\right]$, then

$$
K_{a_{0}, a_{1}, \ldots, a_{m}}(x, t)=-\sum_{k=0}^{m-1} \frac{(m-1) !}{(m-k-1) !} g_{k}(x)\left(a_{k}-t\right)^{m-k-1}
$$

such that

$$
\begin{aligned}
& \int_{x}^{a_{0}} K_{a_{0}, a_{1}, \ldots, a_{m}}(x, t) f^{(m)}(t) \mathrm{d} t \\
& =-\sum_{k=0}^{m-1} \frac{(m-1) !}{(m-k-1) !} g_{k}(x) \int_{x}^{a_{0}}\left(a_{k}-t\right)^{m-k-1} f^{(m)}(t) \mathrm{d} t .
\end{aligned}
$$

We know that the integrands are of type $h(t) f^{(m)}(t)$ with $h(t)$ that does not change sign in $[x, a]$. By means of the first mean value theorem for integrals, we obtain for some $\xi_{0, k} \in[c, d], k=0,1, \ldots, m-1$,

$$
\begin{aligned}
& \int_{x}^{a_{0}} K_{a_{0}, a_{1}, \ldots, a_{m}}(x, t) f^{(m)}(t) \mathrm{d} t \\
& =-\sum_{k=0}^{m-1} \frac{(m-1) !}{(m-k-1) !} g_{k}(x) f^{(m)}\left(\xi_{0, k}\right) \int_{x}^{a_{0}}\left(a_{k}-t\right)^{m-k-1} \mathrm{~d} t .
\end{aligned}
$$

After some calculations, we obtain

$$
\begin{aligned}
& \int_{x}^{a_{0}} K_{a_{0}, a_{1}, \ldots, a_{m}}(x, t) f^{(m)}(t) \mathrm{d} t \\
& =-\sum_{k=0}^{m-1} \frac{(m-1) !}{(m-k-1) !} g_{k}(x) f^{(m)}\left(\xi_{0, k}\right)\left[\left(a_{k}-x\right)^{m-k}-\left(a_{k}-a_{0}\right)^{m-k}\right] .
\end{aligned}
$$

If $t \in\left[a_{0}, a_{1}\right]$, then

$$
\begin{aligned}
K_{a_{0}, a_{1}, \ldots, a_{m}}(x, t) & =-\sum_{k=1}^{m-1} \frac{(m-1) !}{(m-k-1))} g_{k}(x)\left(a_{k}-t\right)^{m-k-1} f^{(m)}(t), \\
& \int_{a_{0}}^{a_{1}} K_{a_{0}, a_{1}, \ldots, a_{m}}(x, t) f^{(m)}(t) \mathrm{d} t \\
& =-\sum_{k=1}^{m-1} \frac{(m-1) !}{(m-k-1) !} g_{k}(x) \int_{a_{0}}^{a_{1}}\left(a_{k}-t\right)^{m-k-1} f^{(m)}(t) \mathrm{d} t .
\end{aligned}
$$

By applying the first mean theorem for the above integrals, we have for some $\xi_{1, k} \in[c, d], k=1, \ldots, m-1$, 
Journal of Mathematics

5

$$
\begin{aligned}
& \int_{a_{0}}^{a_{1}} K_{a_{0}, a_{1}, \ldots, a_{m}}(x, t) f^{(m)}(t) \mathrm{d} t \\
& =-\sum_{k=1}^{m-1} \frac{(m-1) !}{(m-k-1) !} g_{k}(x) f^{(m)}\left(\xi_{1, k}\right) \int_{a_{0}}^{a_{1}}\left(a_{k}-t\right)^{m-k-1} \mathrm{~d} t .
\end{aligned}
$$

After some calculations, we obtain

$$
\begin{aligned}
& \int_{a_{0}}^{a_{1}} K_{a_{0}, a_{1}, \ldots, a_{m}}(x, t) f^{(m)}(t) \mathrm{d} t \\
& =-\sum_{k=1}^{m-1} \frac{(m-1) !}{(m-k-1) !} g_{k}(x) f^{(m)}\left(\xi_{1, k}\right)\left[\left(a_{k}-a_{0}\right)^{m-k}\right. \\
& \left.\quad-\left(a_{k}-a_{1}\right)^{m-k}\right] .
\end{aligned}
$$

If $t \in\left[a_{1}, a_{2}\right]$, then

$$
\begin{aligned}
& K_{a_{0}, a_{1}, \ldots, a_{m}}(x, t)=-\sum_{k=2}^{m-1} \frac{(m-1) !}{(m-k-1) !} g_{k}(x)\left(a_{k}-t\right)^{m-k-1} f^{(m)}(t), \\
& \int_{a_{1}}^{a_{2}} K_{a_{0}, a_{1}, \ldots, a_{m}}(x, t) f^{(m)}(t) \mathrm{d} t \\
&=-\sum_{k=2}^{m-1} \frac{(m-1) !}{(m-k) !} g_{k}(x) \int_{a_{1}}^{a_{2}}\left(a_{k}-t\right)^{m-k-1} f^{(m)}(t) \mathrm{dt} .
\end{aligned}
$$

By applying the first mean theorem for the above integrals, we have for some $\xi_{2, k} \in[c, d], k=2, \ldots, m-1$,

$$
\begin{aligned}
& \int_{a_{1}}^{a_{2}} K_{a_{0}, a_{1}, \ldots, a_{m}}(x, t) f^{(m)}(t) \mathrm{d} t \\
& =-\sum_{k=2}^{m-1} \frac{(m-1) !}{(m-k-1) !} g_{k}(x) f^{(m)}\left(\xi_{2, k}\right) \int_{a_{1}}^{a_{2}}\left(a_{k}-t\right)^{m-k-1} \mathrm{~d} t .
\end{aligned}
$$

After some calculations, we obtain

$$
\begin{aligned}
& \int_{a_{1}}^{a_{2}} K_{a_{0}, a_{1}, \ldots, a_{m}}(x, t) f^{(m)}(t) \mathrm{d} t \\
& =-\sum_{k=2}^{m-1} \frac{(m-1) !}{(m-k) !} g_{k}(x) f^{(m)}\left(\xi_{2, k}\right)\left[\left(a_{k}-a_{1}\right)^{m-k}-\left(a_{k}-a_{2}\right)^{m-k}\right] .
\end{aligned}
$$

In the same way, we have, if $t \in\left[a_{2}, a_{3}\right]$, then for $\xi_{3, k} \in[c, d], k=3, \ldots, m-1$,

$$
\begin{aligned}
& \int_{a_{2}}^{a_{3}} K_{a_{0}, a_{1}, \ldots, a_{m}}(x, t) f^{(m)}(t) \mathrm{d} t \\
& =-\sum_{k=3}^{m-1} \frac{(m-1) !}{(m-k) !} g_{k}(x) f^{(m)}\left(\xi_{3, k}\right)\left[\left(a_{k}-a_{2}\right)^{m-k}-\left(a_{k}-a_{3}\right)^{m-k}\right] .
\end{aligned}
$$

If $t \in\left[a_{m-3}, a_{m-2}\right]$, then for $\xi_{m-2, k} \in[c, d], k=m-2, m-$ 1 ,

$$
\begin{aligned}
& \int_{a_{m-3}}^{a_{m}-2} K_{a_{0}, a_{1}, \ldots, a_{m}}(x, t) f^{(m)}(t) \mathrm{d} t \\
& =-\sum_{k=m-2}^{m-1} \frac{(m-1) !}{(m-k) !} g_{k}(x) f^{(m)}\left(\xi_{m-2, k}\right)\left[\left(a_{k}-a_{m-3}\right)^{m-k}\right. \\
& \left.\quad-\left(a_{k}-a_{m-2}\right)^{m-k}\right] .
\end{aligned}
$$

If $t \in\left[a_{m-2}, a_{m-1}\right]$, then for $\xi_{m-1, k} \in[c, d], k=m-1$,

$$
\begin{aligned}
& \int_{a_{m-2}}^{a_{m-1}} K_{a_{0}, a_{1}, \ldots, a_{m}}(x, t) f^{(m)}(t) \mathrm{d} t \\
& =-\sum_{k=m-1}^{m-1} \frac{(m-1) !}{(m-k) !} g_{k}(x) f^{(m)}\left(\xi_{m-1, k}\right)\left[\left(a_{k}-a_{m-2}\right)^{m-k}\right. \\
& \left.\quad-\left(a_{k}-a_{m-1}\right)^{m-k}\right] .
\end{aligned}
$$

By definition of (19), $K_{a_{0}, a_{1}, \ldots, a_{m}}(x, t)$ of (18) is zero as $a_{m-1} \leq t \leq a_{m}$. Substituting into (26) the left-hand sides of (30), (33), (36), (37), (38), and (39) by their respective righthand sides, we finally get the expression as follows:

$$
\begin{aligned}
R_{m} & {\left[f ; a_{0}, a_{1}, \ldots, a_{m}\right](x, t) } \\
= & -\sum_{k=0}^{m-1} \frac{1}{(m-k) !} g_{k}(x) f^{(m)}\left(\xi_{0, k}\right)\left[\left(a_{k}-x\right)^{m-k}-\left(a_{k}-a_{0}\right)^{m-k}\right] \\
& -\sum_{k=1}^{m-1} \frac{1}{(m-k) !} g_{k}(x) f^{(m)}\left(\xi_{1, k}\right)\left[\left(a_{k}-a_{0}\right)^{m-k}-\left(a_{k}-a_{1}\right)^{m-k}\right] \\
& -\cdots \\
& -\sum_{k=m-2}^{m-1} \frac{1}{(m-k) !} g_{k}(x) f^{(m)}\left(\xi_{m-2, k}\right)\left[\left(a_{k}-a_{m-3}\right)^{m-k}\right. \\
& \left.-\left(a_{k}-a_{m-2}\right)^{m-k}\right] \\
& -\sum_{k=m-1}^{m-1} \frac{1}{(m-k) !} g_{k}(x) f^{(m)}\left(\xi_{m-1, k}\right)\left[\left(a_{k}-a_{m-2}\right)^{m-k}\right. \\
& \left.-\left(a_{k}-a_{m-1}\right)^{m-k}\right] .
\end{aligned}
$$

In order to obtain the desired bounds (24), we have the following inequation for $k=0,1, m$ :

$$
\begin{aligned}
\left|g_{k}(x)\right| & =\left|\int_{a_{0}}^{x} \mathrm{~d} t_{1} \int_{a_{1}}^{t_{1}} \mathrm{~d} t_{2}, \ldots, \int_{a_{k-1}}^{t_{k-1}} \mathrm{~d} t_{k}\right| \\
& \leq\left(a_{m-1}-x\right)^{k}
\end{aligned}
$$

where the inequality follows from (10): 


$$
\begin{aligned}
\left|g_{0}(x)\right| & =1 \leq\left(a_{m-1}-x\right)^{0}, \\
\left|g_{1}(x)\right| & =\left|x-x_{0}\right| \leq\left(a_{m-1}-x\right), \\
\left|g_{k}(x)\right| & =\left|\int_{a_{0}}^{x} \mathrm{~d} t_{1} \int_{a_{1}}^{t_{1}} \mathrm{~d} t_{2}, \ldots, \int_{a_{k-1}}^{t_{k-1}} \mathrm{~d} t_{k}\right| \\
& \leq\left|\int_{a_{0}}^{x} \mathrm{~d} t_{1} \int_{a_{1}}^{x} \mathrm{~d} t_{2}, \ldots, \int_{a_{k-1}}^{x} \mathrm{~d} t_{k}\right| \\
& \leq\left|\left(x-a_{0}\right)\left(x-a_{1}\right), \ldots,\left(x-a_{k-1}\right)\right| \\
& \leq\left(a_{m-1}-x\right)^{k}, \quad(k=2,3, \ldots, m) .
\end{aligned}
$$

Finally, we have, after some calculations,

$$
\begin{aligned}
& \left|R_{m}\left[f ; a_{0}, a_{1}, \ldots, a_{m}\right]\right| \\
& \leq\left\|f^{(m)}\right\|_{\infty}\left[( a _ { m - 1 } - x ) ^ { m } \left(\sum_{k=0}^{m-1} \frac{1}{(m-k) !}\right.\right. \\
& \left.\left.+\sum_{k=1}^{m-1} \frac{1}{(m-k) !}+\cdots+\sum_{k=m-2}^{m-1} \frac{1}{(m-k) !}+\sum_{k=m-1}^{m-1} \frac{1}{(m-k) !}\right)\right] \\
& \leq\left[1+\left(\sum_{k=0}^{m-1}+\sum_{k=1}^{m-1}+\cdots+\sum_{k=m-2}^{m-1}\right) \frac{1}{(m-k) !}\right]\left\|f^{(m)}\right\|_{\infty}\left(a_{m-1}-x\right)^{m} .
\end{aligned}
$$

Similarly, the remaining expressions of (24) could be proved.

Since the algebraic degree of exactness of the operator $P_{m}\left[f ; a_{0}, a_{1}, \ldots, a_{m}\right]$ is equal to $m$, the following result can be given in an analogous manner.
Theorem 4. Given $f \in C^{m+1}[c, d]$ and $x \in[c, d]$, for the remainder (16), we have

$$
\begin{aligned}
& \left|R_{m}\left[f ; a_{0}, a_{1}, \ldots, a_{m}\right](x)\right| \\
& \leq \begin{cases}C(m+1)\left\|f^{(m+1)}\right\|_{\infty}\left(a_{m}-x\right)^{m+1}, & c \leq x \leq a_{0}, \\
C(m+1)\left\|f^{(m+1)}\right\|_{\infty}\left(a_{m}-a_{0}\right)^{m+1}, & a_{0} \leq x \leq a_{m}, \\
C(m+1)\left\|f^{(m+1)}\right\|_{\infty}\left(x-a_{0}\right)^{m+1}, & a_{m} \leq x \leq d,\end{cases}
\end{aligned}
$$

where $C(m)$ is defined in (25).

\section{The Abel-Goncharov Type Multiquadric Quasi-Interpolants}

3.1. A Kind of Abel-Goncharov Type Multiquadric QuasiInterpolants. For $N+1$ pairwise distinct points $x_{i} \in[a, b], 0 \leq i \leq N$, and the values $f^{(i)}\left(x_{i}\right), i=0,1, \ldots, N$, we attach to each node $x_{i}, i=0,1, \ldots, N$, a set of nodes $X_{i m}, m \in \mathbb{N}, m \in N, i=0,1, \ldots, N$, defined by

$$
X_{i m}=\left\{x_{i}, x_{i+1}, \ldots, x_{i+m}\right\}, i=0,1, \ldots, N,
$$

where $x_{N+j}=x_{N-m+j-1}, j=1, \ldots, m$. By using each set of nodes $X_{i m}, i=0,1, \ldots, N$, the Abel-Goncharov interpolation operator in (8) can be denoted by $P_{m}^{i}, i=0,1, \ldots, N$, i.e.,

$$
\left(P_{m}^{i}\left[f ; x_{i}, x_{i+1}, \ldots, x_{i+m}\right]\right)^{(k)}=f^{(k)}\left(x_{k}\right), \quad i \leq k \leq i+m, 0 \leq i \leq N,
$$

where we have

$$
\left(P_{m}^{i}\left[f ; x_{i}, x_{i+1}, \ldots, x_{i+m}\right]\right)(x)=\sum_{j=i}^{i+m} g_{j-i}^{\langle i\rangle}(x) f^{(j-i)}\left(x_{j}\right), \quad i=0,1, \ldots, N
$$

with the Goncharov polynomials

$$
\begin{aligned}
g_{0}^{\langle i\rangle}(x) & =1, \\
g_{1}^{\langle i\rangle}(x) & =x-x_{i}, \\
& \ldots, \\
g_{k}^{\langle i\rangle}(x) & =\frac{1}{k !}\left[x^{k}-\sum_{j=0}^{k-1} g_{j}^{\langle i\rangle}(x)\left(\begin{array}{c}
k \\
j
\end{array}\right)\right], \quad k=2, \ldots, m .
\end{aligned}
$$

Theorem 5. The Abel-Goncharov interpolants $P_{m}^{i}, i=0,1$, $\ldots, N$, possess the degree of exactness $m$.

Proof. The proof is given similarly to that of Theorem 1 .

Furthermore, we recall the well-known multiquadric quasi-interpolation operator $\mathscr{L}_{B}$, defined by Beatson and Powell in [6], as follows:

$$
\left(\mathscr{L}_{B} f\right)(x)=f\left(x_{0}\right) \psi_{0}(x)+\sum_{i=1}^{N-1} f\left(x_{i}\right) \psi_{i}(x)+f\left(x_{N}\right) \psi_{N}(x), \quad x \in I,
$$


where

$$
\begin{aligned}
\psi_{0}(x) & =\frac{1}{2} c^{2} \int_{-\infty}^{x_{0}} \frac{1}{\left[(x-t)^{2}+c^{2}\right]^{3 / 2}} \mathrm{~d} t+\frac{1}{2} c^{2} \int_{x_{0}}^{x_{1}} \frac{\left(x_{1}-t\right) /\left(x_{1}-x_{0}\right)}{\left[(x-t)^{2}+c^{2}\right]^{3 / 2}} \mathrm{~d} t \\
& =\frac{1}{2}+\frac{\phi_{1}(x)-\phi_{0}(x)}{2\left(x_{1}-x_{0}\right)} \\
\psi_{N}(x) & =\frac{1}{2} c^{2} \int_{x_{N}}^{\infty} \frac{1}{\left[(x-t)^{2}+c^{2}\right]^{3 / 2}}+\frac{1}{2} c^{2} \int_{x_{N-1}}^{x_{N}} \frac{\left(t-x_{N-1}\right) /\left(x_{N}-x_{N-1}\right)}{\left[(x-t)^{2}+c^{2}\right]^{3 / 2}} \mathrm{~d} t \\
& =\frac{1}{2}-\frac{\phi_{N}(x)-\phi_{N-1}(x)}{2\left(x_{N}-x_{N-1}\right)}, \\
\psi_{i}(x) & =\frac{1}{2} c^{2} \int_{x_{i-1}}^{x_{i+1}} \frac{B_{i}(t)}{\left[(x-t)^{2}+c^{2}\right]^{3 / 2}} \mathrm{~d} t \\
& =\frac{\phi_{i+1}(x)-\phi_{i}(x)}{2\left(x_{i+1}-x_{i}\right)}-\frac{\phi_{i}(x)-\phi_{i-1}(x)}{2\left(x_{i}-x_{i-1}\right)}
\end{aligned}
$$

for $i=1,2, \ldots, N-1$, where $B_{i}(t)$ is the piecewise linear hat function with the knots $\left\{x_{i-1}, x_{i}, x_{i+1}\right\}$ and satisfies $B_{i}\left(x_{i}\right)=1$. The quasi-interpolation operator $\mathscr{L}_{B}$ reproduces constants, i.e.,

$$
\sum_{i=0}^{N} \psi_{i}(x)=1
$$

By combining the quasi-interpolation operator $\mathscr{L}_{B}$ with Abel-Goncharov interpolation polynomials, we construct a kind of Abel-Goncharov type multiquadric quasi-interpolation operator $\mathscr{L}_{m}^{A G}$ as follows:

$$
\left(\mathscr{L}_{m}^{A G} f\right)(x)=\sum_{i=0}^{N} \psi_{i}(x) P_{m}^{i}\left[f ; x_{i}, x_{i+1}, \ldots, x_{i+m}\right](x), \quad x \in I
$$

where $P_{m}^{i}\left[f ; x_{i}, x_{i+1}, \ldots, x_{i+m}\right](x), i=0,1, \ldots, N$, is the Abel-Goncharov interpolation polynomials defined in (47) and $x_{N+j}=x_{N-m+j-1}, j=1,2, \ldots, m$.

Theorem 6. The operator $\mathscr{L}_{m}^{A G} f$ reproduces all univariate polynomials of degree $\leq m$.

Proof. $\mathscr{L}_{m}^{A G} p=p$ is proved from the following result:

$$
\begin{aligned}
\sum_{i=0}^{N} \psi_{i}(x) & =1, \\
P_{m}^{i}\left[p ; x_{i}, x_{i+1}, \ldots, x_{i+m}\right] & =p, \quad p \in \mathbb{P}_{m},
\end{aligned}
$$

for $i=0,1, \ldots, N$.

3.2. The Convergence Rate of the Operator. In order to give the convergence rate of the operator $\mathscr{L}_{m}^{A G}$, we apply the following notations:

$$
\begin{aligned}
I_{\rho}(x) & =[x-\rho, x+\rho], \quad \rho>0, \\
r & =\inf \left\{\rho>0: \forall x \in I, I_{\rho}(x) \cap X \neq \varnothing\right\}, \\
M & =\max _{x \in I} \#\left(I_{r}(x) \cap X\right),
\end{aligned}
$$

for $X=\left\{x_{0}, x_{1}, \ldots, x_{N}\right\}$, where \#(.) denotes the cardinality function. Thus, we can obtain $2 r=\max \left\{\left|x_{1}-x_{0}\right|\right.$, $\left.\left|x_{2}-x_{1}\right|, \ldots,\left|x_{N}-x_{N-1}\right|\right\}$, and $M$ denotes the maximum number of points from $X$ contained in an interval $I_{r}(x)$. Then, we provide the following error estimates.

Theorem 7. Suppose that $c$ satisfies

$$
c \leq D r^{l},
$$

where $D$ is a positive constant and $l$ is a positive integer. If $f(x) \in C^{m}(I)$, then

$$
\left\|\left(\mathscr{L}_{m}^{A G} f\right)(x)-f(x)\right\|_{\infty} \leq K M\left\|f^{(m)}\right\|_{\infty} \mathscr{E}_{l, m}(r),
$$


where

$$
\mathscr{E}_{l, m}(r)= \begin{cases}r|\ln r|, & m=1, l=1, \\ r, & m=1, l>1, \\ r^{m}, & m>1, m \leq 2 l-l, \\ r^{2 l-1}, & m>1, m>2 l-l,\end{cases}
$$

and $K$ is a positive constant independent of $x$ and $X$.

$$
\begin{aligned}
d\left[x_{i}, x_{i+1}, \ldots, x_{i+m}\right] & = \begin{cases}x_{i+m-1}-x, & x \leq x_{i}, \\
x_{i+m-1}-x_{i}, & x_{i} \leq x \leq x_{i+m-1}, \\
x-x_{i}, & x_{i+m-1} \leq x,\end{cases} \\
d^{m}\left[x_{i}, x_{i+1}, \ldots, x_{i+m}\right] & =\left(d\left[x_{i}, x_{i+1}, \ldots, x_{i+m}\right]\right)^{m} .
\end{aligned}
$$

Based on (24), (47), and (52), we can give

Proof. Let $x_{i}, x_{i+1}, \ldots, x_{i+m} \in I$ be fixed, $i=0,1, \ldots, N$, $m \in \mathbb{N}, x_{i}<x_{i+1}, \ldots, x_{i+m}$; for each $x \in I$, we set

$$
\begin{aligned}
\left|\left(L_{m}^{A G} f\right)(x)-f(x)\right| & =\left|\sum_{i=0}^{N} \psi_{i}(x) P_{m}^{i}\left[f ; x_{i}, x_{i+1}, \ldots, x_{i+m}\right](x)-f(x)\right| \\
& \leq \sum_{i=0}^{N} \psi_{i}(x)\left|P_{m}^{i}\left[f ; x_{i}, x_{i+1}, \ldots, x_{i+m}\right](x)-f(x)\right| \\
& \leq C(m)\left\|f^{(m)}\right\|_{\infty} \mathcal{S}_{l, m}(x),
\end{aligned}
$$

where

$$
\mathcal{S}_{l, m}(x)=\sum_{i=0}^{N} \psi_{i}(x) d^{m}\left[x_{i}, x_{i+1}, \ldots, x_{i+m}\right](x) .
$$

Assume that

$$
\begin{aligned}
n & =\left[\frac{x_{N}-x_{0}}{2 r}\right]+1, \\
Q_{\rho}(u) & =(u-\rho, u+\rho], \quad u \in I, \rho>0, \\
T_{j} & =Q_{r}(x-2 r j) \cup Q_{r}(x+2 r j), \quad j=0, \ldots, n,
\end{aligned}
$$

$$
\begin{aligned}
(2 j-1) r & \leq\left|x-x_{i}\right| \leq(2 j+1) r \\
(2(j-1)-1) r & \leq\left|x-\tau_{i}\right| \leq(2(j+1)+1) r, \quad \text { for } \tau_{i} \in\left[x_{i-1}, x_{i+1}\right], \\
\left|d\left[x_{i}, x_{i+1}, \ldots, x_{i+m}\right](x)\right| & \leq(2(j+m-1)+1) r .
\end{aligned}
$$

We have from the definition of $M$

$$
\begin{aligned}
\psi_{0}(x) & \leq \frac{1}{2} c^{2} \int_{-\infty}^{x_{0}} \frac{1}{|x-t|^{3}} \mathrm{~d} t+\frac{1}{2} c^{2} \frac{1}{\left[\left(x-\tau_{0}\right)^{2}+c^{2}\right]^{3 / 2}} \int_{x_{0}}^{x_{1}} \frac{x_{1}-t}{x_{1}-x_{0}} \mathrm{~d} t \\
& \leq \frac{1}{4} c^{2}\left|x-x_{0}\right|^{-2}+\frac{1}{4} c^{2}\left(x_{1}-x_{0}\right)\left|x-\tau_{0}\right|^{-3} \\
& \leq \frac{1}{4} c^{2}\left[(2 j-1)^{-2} r^{-2}+2(2 j-3)^{-3} r^{-2}\right] \\
& \leq c^{2} r^{-2}(2 j-3)^{-2},
\end{aligned}
$$$$
\begin{aligned}
& 1 \leq \#\left(X \cap T_{0}\right) \leq M, \\
& 1 \leq \#\left(X \cap T_{j}\right) \leq 2 M, \quad j=1,2, \ldots, n .
\end{aligned}
$$

When $x_{0} \in T_{j}, j=2,3, \ldots, n$, we have, after some calculations, 
where $\tau_{0} \in\left[x_{0}, x_{1}\right]$. When $x_{N} \in T_{j}, j=2,3, \ldots, n$, we get in an analogous manner

$$
\psi_{N}(x) \leq c^{2} r^{-2}(2 j-3)^{-2} \text {. }
$$

When $x_{i}(i=1, \ldots, N-1) \in T_{j}, j=2,3, \ldots, n$, we also get

$$
\begin{aligned}
\psi_{i}(x) & \leq \frac{1}{2} c^{2} \frac{1}{\left[\left(x-\tau_{i}\right)^{2}+c^{2}\right]^{3 / 2}} \int_{x_{i-1}}^{x_{i+1}} B_{i}(t) \mathrm{d} t \\
& \leq \frac{1}{4} c^{2}\left(x_{i+1}-x_{i-1}\right)\left|x-\tau_{i}\right|^{-3} \\
& \leq c^{2} r^{-2}(2 j-3)^{-2}
\end{aligned}
$$

where $\tau_{i} \in\left[x_{i-1}, x_{i+1}\right]$. Then, we have

$$
\begin{aligned}
\mathcal{S}_{l, m}(x) \leq & \sum_{x_{i} \in T_{0}, T_{1}} \psi_{i}(x) d^{m}\left[x_{i}, x_{i+1}, \ldots, x_{i+m}\right](x) \\
& +\sum_{j=2}^{n} \sum_{x_{i} \in T_{j}} \psi_{i}(x) d^{m}\left[x_{i}, x_{i+1}, \ldots, x_{i+m}\right](x) \\
\leq & \sum_{x_{i} \in T_{0}, T_{1}} d^{m}\left[x_{i}, x_{i+1}, \ldots, x_{i+m}\right](x) \\
& +\sum_{j=2}^{n} \sum_{x_{i} \in T_{j}} \psi_{i}(x) d^{m}\left[x_{i}, x_{i+1}, \ldots, x_{i+m}\right](x) \\
\leq & M[(2 m-1) r]^{m}+2 M[(2 m+1) r]^{m} \\
& +2 M \sum_{j=2}^{n} c^{2} r^{-2}(2 j-3)^{-2}[(2 j+2 m-1) r]^{m} \\
\leq & 2 M\left[((2 m+1) r)^{m}+((2 m+1) r)^{m}\right. \\
& \left.+c^{2} r^{m-2} \sum_{j=2}^{n}(2 j-3)^{-2}(2 j+2 m-1)^{m}\right] \\
\leq & 2 M(2 m+1)^{m}\left(2 r^{m}+D^{2} r^{m+2 l-2} \sum_{j=1}^{n} j^{m-2}\right)
\end{aligned}
$$

where the last inequality follows the form

$$
\begin{cases}2 j-3 \geq j, & j=3,4, \ldots, n, \\ 2 j+2 m-1 \leq(2 m+1) j, & j=1,2, \ldots, n, \\ c \leq D r^{l} & \end{cases}
$$

Case 1. $(m=1)$.

(i) Let $\quad l=1 . \quad$ Then, $\quad 2 r^{m}+D^{2} r^{m+2 l-2} \quad \sum_{j=1}^{n} j^{m-2}$ $=\mathcal{O}(r|\ln r|)$.

(ii) Let $l>1$. Then, $2 r^{m}+D^{2} r^{m+2 l-2} \sum_{j=1}^{n} j^{m-2}=\mathcal{O}(r)$.

Case 2. $(m>1)$.

(i) Let $m \leq 2 l-1$. Then, $2 r^{m}+D^{2} r^{m+2 l-2} \sum_{j=1}^{n} j^{m-2}$ $=\mathscr{O}\left(r^{m}\right)$. (ii) Let $m>2 l-1$. Then, $2 r^{m}+D^{2} r^{m+2 l-2} \sum_{j=1}^{n} j^{m-2}=$ $\mathcal{O}\left(r^{2 l-1}\right)$.

In an analogous manner, we can obtain the desired error estimation as follows:

Theorem 8. Let $c$ satisfy

$$
c \leq D r^{l}
$$

where $D$ is a positive constant and $l$ is a positive integer. If $f(x) \in C^{m+1}(I)$, then

$$
\left\|\left(\mathscr{L}_{m}^{A G} f\right)(x)-f(x)\right\|_{\infty} \leq K^{\prime} M\left\|f^{(m+1)}\right\|_{\infty} \mathscr{E}_{l, m}^{\prime}(r),
$$

where

$$
\mathscr{E}_{l, m}^{\prime}(r)= \begin{cases}r^{2 l-1}, & m+1 \geq 2 l-1, \\ r^{m+1}, & m+1 \leq 2 l-1,\end{cases}
$$

and $K^{\prime}$ is a positive constant independent of $x$ and $X$.

\section{Numerical Examples}

In order to investigate the accuracy of our operators, we use the following functions on the interval $[0,1][33]$.

$$
\begin{aligned}
\text { Saddle } f_{1} & =\frac{1.25}{6+6(3 x-1)^{2}}, \\
\text { Sphere } f_{2} & =\frac{\sqrt{64-81(x-0.5)^{2}}}{9}-0.5, \\
\text { Cliff } f_{3} & =\frac{\tanh (-9 x+1)}{2}+0.5, \\
\text { Gentle } f_{4} & =\frac{\exp \left(-(81 / 16)(x-0.5)^{2}\right)}{3}, \\
\text { Steep } f_{5} & =\frac{\exp \left(-(81 / 4)(x-0.5)^{2}\right)}{3},
\end{aligned}
$$

Exponential $f_{6}=0.75 \exp \left(-\frac{(9 x-2)^{2}}{4}\right)+0.75 \exp \left(-\frac{(9 x+1)^{2}}{49}\right)$

$$
+0.5 \exp \left(-\frac{(9 x-7)^{2}}{4}\right)+0.2 \exp \left(-(9 x-4)^{2}\right) \text {. }
$$

For each function $f_{i}, i=1,2, \ldots, 6$, we will compare the numerical results of our new operator $\mathscr{L}_{m}^{A G}$ with the known operator $\mathscr{L}_{H_{2 m-1}}[10]$ as $c=r^{l}$. We consider a uniform grid of 17 points for $\mathscr{L}_{1}^{A G}$ and $\mathscr{L}_{H_{1}}$, grid of 11 points for $\mathscr{L}_{2}^{A G}$, and grid of 8 points for $\mathscr{L}_{\mathrm{H}_{3}}$ on the interval $[0,1]$. In order to obtain the estimation as accurate as possible, we calculated the approximated functions at the points $(i / 101), i=1,2$, $\ldots, 100$. Tables $1-6$ present the mean and maximum errors, computed for different values of parameters $l$ and $m$. These results show that the approximations of Abel-Goncharov multiquadric quasi-interpolants $\mathscr{L}_{m}^{A G}$ are comparable with that of the multiquadric quasi-interpolants $\mathscr{L}_{H_{2 m-1}}$. 
Table 1: Saddle.

\begin{tabular}{lcccc}
\hline & $\mathscr{L}_{H_{2 m-1}} f_{1}$ & & $\mathscr{L}_{m}^{A G} f_{1}$ & $\varepsilon_{\text {mean }}$ \\
$(l, m)$ & $\varepsilon_{\text {mean }}$ & $\varepsilon_{\max }$ & 0.000476 & 0.002273 \\
$(2,1)$ & 0.000398 & 0.001875 & 0.001960 & 0.009792 \\
$(2,2)$ & 0.000336 & 0.002426 & 0.000473 & 0.002270 \\
$(3,1)$ & 0.000395 & 0.001873 & 0.001944 & 0.009775 \\
$(3,2)$ & 0.000330 & 0.002429 & 0.000473 & 0.002270 \\
$(4,1)$ & 0.000395 & 0.001873 & 0.001944 & 0.009775 \\
$(4,2)$ & 0.000330 & 0.002429 & & \\
\hline
\end{tabular}

TABle 2: Sphere.

\begin{tabular}{lccrr}
\hline & $\mathscr{L}_{H_{2 m-1}} f_{2}$ & & & $\mathscr{L}_{m}^{A G} f_{2}$ \\
$(l, m)$ & $\varepsilon_{\text {mean }}$ & $\varepsilon_{\max }$ & $\varepsilon_{\text {mean }}$ & 0.002686 \\
\hline$(2,1)$ & 0.000449 & 0.000895 & 0.000526 & 0.002127 \\
$(2,2)$ & 0.000035 & 0.000134 & 0.000394 & 0.002687 \\
$(3,1)$ & 0.000445 & 0.000896 & 0.000522 & 0.002128 \\
$(3,2)$ & 0.000032 & 0.000132 & 0.000392 & 0.002687 \\
$(4,1)$ & 0.000445 & 0.000896 & 0.000522 & 0.002128 \\
$(4,2)$ & 0.000032 & 0.000132 & 0.000392 & \\
\hline
\end{tabular}

Table 3: Cliff.

\begin{tabular}{lccrr}
\hline & $\mathscr{L}_{H_{2 m-1}} f_{3}$ & & & $\mathscr{L}_{m}^{A G} f_{3}$ \\
$(l, m)$ & $\varepsilon_{\text {mean }}$ & $\varepsilon_{\max }$ & $\varepsilon_{\text {mean }}$ & 0.029450 \\
\hline$(2,1)$ & 0.001952 & 0.014873 & 0.002110 & 0.110462 \\
$(2,2)$ & 0.004444 & 0.050405 & 0.010699 & 0.029426 \\
$(3,1)$ & 0.001943 & 0.014863 & 0.002101 & 0.110363 \\
$(3,2)$ & 0.004342 & 0.050377 & 0.010604 & 0.029426 \\
$(4,1)$ & 0.001943 & 0.014863 & 0.002101 & 0.110363 \\
$(4,2)$ & 0.004342 & 0.050377 & 0.010604 & \\
\hline
\end{tabular}

TABLe 4: Gentle.

\begin{tabular}{lcccc}
\hline & $\mathscr{L}_{H_{2 m-1}} f_{4}$ & & $\mathscr{L}_{m}^{A G} f_{4}$ & $\varepsilon_{\text {mean }}$ \\
$(l, m)$ & $\varepsilon_{\text {mean }}$ & $\varepsilon_{\max }$ & 0.000654 & 0.001975 \\
$(2,1)$ & 0.000545 & 0.001645 & 0.002037 & 0.005950 \\
$(2,2)$ & 0.000185 & 0.000741 & 0.000650 & 0.001975 \\
$(3,1)$ & 0.000542 & 0.001642 & 0.002021 & 0.005949 \\
$(3,2)$ & 0.000181 & 0.000739 & 0.000650 & 0.001975 \\
$(4,1)$ & 0.000542 & 0.001642 & 0.002021 & 0.005949 \\
$(4,2)$ & 0.000181 & 0.000739 & & \\
\hline
\end{tabular}

Table 5: Steep.

\begin{tabular}{lcccc}
\hline & $\mathscr{L}_{H_{2 m-1}} f_{5}$ & & $\mathscr{L}_{m}^{A G} f_{5}$ & $\varepsilon_{\text {mean }}$ \\
$(l, m)$ & $\varepsilon_{\text {mean }}$ & $\varepsilon_{\text {max }}$ & 0.002150 & 0.008659 \\
$(2,1)$ & 0.001700 & 0.006483 & 0.012294 & 0.037863 \\
$(2,2)$ & 0.001917 & 0.009080 & 0.002138 & 0.008651 \\
$(3,1)$ & 0.001692 & 0.006477 & 0.012200 & 0.037811 \\
$(3,2)$ & 0.001893 & 0.009100 & 0.002138 & 0.008651 \\
$(4,1)$ & 0.001692 & 0.006477 & 0.012200 & 0.037811 \\
$(4,2)$ & 0.001893 & 0.009100 & & \\
\hline
\end{tabular}


TABle 6: Exponential.

\begin{tabular}{lcccc}
\hline & $\mathscr{L}_{H_{2 m-1}} f_{6}$ & & \multicolumn{2}{c}{$\mathscr{L}_{m}^{A G} f_{6}$} \\
$(l, m)$ & $\varepsilon_{\text {mean }}$ & $\varepsilon_{\max }$ & $\varepsilon_{\text {mean }}$ & $\varepsilon_{\max }$ \\
\hline$(2,1)$ & 0.004663 & 0.015048 & 0.006739 & 0.026089 \\
$(2,2)$ & 0.010839 & 0.035808 & 0.041908 & 0.152439 \\
$(3,1)$ & 0.004645 & 0.015044 & 0.006714 & 0.026077 \\
$(3,2)$ & 0.010731 & 0.035689 & 0.041734 & 0.152347 \\
$(4,1)$ & 0.004645 & 0.015044 & 0.006714 & 0.026077 \\
$(4,2)$ & 0.010731 & 0.035689 & 0.041733 & 0.152347 \\
\hline
\end{tabular}

\section{Conclusions}

In this study, by combing multiquadric quasi-interpolant $\mathscr{L}_{B}$ with the Abel-Goncharov univariate operator, we construct a kind of Abel-Goncharov multiquadric quasiinterpolants $\mathscr{L}_{m}^{A G} f$. Meanwhile, we have also proven that the operators $\mathscr{L}_{m}^{A G} f$ possess the $m^{\text {th }}$ degree polynomial reproduction property and good convergence capacity, so that it is convenient for people in various applications. Moreover, the associated algorithm is easily implemented.

In our future work, the univariate Abel-Goncharov type multiquadric quasi-interpolants can be extended to the multivariate case. Moreover, we can also apply the operators to fit scattered data.

\section{Data Availability}

The data used to support the findings of this study are available from the corresponding author upon request.

\section{Conflicts of Interest}

The authors declare that they have no conflicts of interest.

\section{Acknowledgments}

The work was supported by the Open Project of Key Laboratory of Symbolic Computation and Knowledge Engineering of Ministry of Education, Jilin University (Grant no. 93K172019K13).

\section{References}

[1] R. L. Hardy, "Multiquadric equations of topography and other irregular surfaces," Journal of Geophysical Research, vol. 76, no. 8, pp. 1905-1915, 1971.

[2] M. D. Buhmann, "Multivariate interpolation in odd-dimensional Euclidean spaces using multiquadrics," Constructive Approximation, vol. 6, no. 1, pp. 21-34, 1990.

[3] M. D. Buhmann, "Multivariate cardinal interpolation with radial-basis functions," Constructive Approximation, vol. 6, no. 3, pp. 225-255, 1990.

[4] R. Fkanke, "Scattered data interpolation: tests of some methods," Mathematics of Computation, vol. 38, pp. 181-200, 1982.

[5] C. A. Micchelli, "Interpolation of scattered data: distance matrices and conditionally positive definite functions," Constructive Approximation, vol. 2, no. 1, pp. 11-22, 1986.
[6] R. K. Beatson and M. J. D. Powell, "Univariate multiquadric approximation: quasi-interpolation to scattered data," Constructive Approximation, vol. 8, no. 3, pp. 275-288, 1992.

[7] Z. Wu and S. Robert, "Shape preserving properties and convergence of univariate multiquadric quasi-interpolation," Acta Mathematicae Applicatae Sinica, vol. 10, no. 4, pp. 441-446, 1994.

[8] L. Ling, "A univariate quasi-multiquadric interpolationwith better smoothness," Computers \& Mathematics with Applications, vol. 48, no. 5-6, pp. 897-912, 2004.

[9] R. Feng and X. Zhou, "A kind of multiquadric quasi-interpolation operator satisfying any degree polynomial reproduction property to scattered data," Journal of Computational and Applied Mathematics, vol. 235, no. 5, pp. 1502-1514, 2011.

[10] R.-H. Wang, M. Xu, and Q. Fang, "A kind of improved univariate multiquadric quasi-interpolation operators," Computers \& Mathematics with Applications, vol. 59, no. 1, pp. 451-456, 2010.

[11] G. J. Lidstone, "Notes on the extension of Aitken's theorem (for polynomial interpolation) to the Everett types," Proceedings of the Edinburgh Mathematical Society, vol. 2, pp. 16-19, 1929.

[12] F. A. Costabile and F. Dell'Accio, "Lidstone approximation on the triangle," Applied Numerical Mathematics, vol. 52, no. 4, pp. 339-361, 2005.

[13] T. Catinas, "The Lidstone interpolation on tetrahedron," Journal of Applied Functional Analysis, vol. 4, pp. 425-439, 2006.

[14] R. Wu, H. Li, and T. Wu, "Univariate Lidstone-type multiquadric quasi-interpolants," Computational and Applied Mathematics, vol. 39, no. 3, p. 141, 2020.

[15] R. Chen and $\mathrm{Z}$. Wu, "Applying multiquadric quasi-interpolation to solve Burgers' equation," Applied Mathematics and Computation, vol. 172, no. 1, pp. 472-484, 2006.

[16] R. Chen and Z. Wu, "Solving partial differential equation by using multiquadric quasi-interpolation," Applied Mathematics and Computation, vol. 186, no. 2, pp. 1502-1510, 2007.

[17] Y. C. Hon and Z. Wu, "A quasi-interpolation method for solving stiff ordinary differential equations," International Journal for Numerical Methods in Engineering, vol. 48, no. 8, pp. 1187-1197, 2000.

[18] Z. Wu, "Dynamically knots setting in meshless method for solving time dependent propagations equation," Computer Methods in Applied Mechanics and Engineering, vol. 193, no. 12-14, pp. 1221-1229, 2004.

[19] Z. Wu, "Dynamical knot and shape parameter setting for simulating shock wave by using multi-quadric quasi-interpolation," Engineering Analysis with Boundary Elements, vol. 29, no. 4, pp. 354-358, 2005.

[20] Z. Wu and S. Zhang, "Conservative multiquadric quasi-interpolation method for Hamiltonian wave equations," Engineering Analysis with Boundary Elements, vol. 37, no. 7-8, pp. 1052-1058, 2013.

[21] W. Bao and Y. Song, "Multiquadric quasi-interpolation methods for solving partial differential algebraic equations," Numerical Methods for Partial Differential Equations, vol. 30, no. 1, pp. 95-119, 2014.

[22] F. Gao and C. Chi, "Numerical solution of nonlinear Burgers' equation using high accuracy multi-quadric quasi-interpolation," Applied Mathematics and Computation, vol. 229, pp. 414-421, 2014.

[23] H.-Y. Wu and Y. Duan, "Multi-quadric quasi-interpolation method coupled with FDM for the Degasperis-Procesi 
equation," Applied Mathematics and Computation, vol. 274, pp. 83-92, 2016.

[24] S. Zhang, C. Zhu, and Q. Gao, "Numerical solution of highdimensional shockwave equations by bivariate multi-quadric quasi-interpolation," Mathematics, vol. 7, no. 8, p. 734, 2019.

[25] S. Zhang and L. Zhang, "Symplectic multiquadric quasi-interpolation approximations of $\mathrm{KdV}$ equation," Filomat, vol. 32, no. 15, pp. 5161-5171, 2018.

[26] S. Zhang, H. Yang, and Y. Yang, "A multiquadric quasi-interpolations method for CEV option pricing model," Journal of Computational and Applied Mathematics, vol. 347, pp. 1-11, 2019.

[27] F. A. M. Ali, S. A. Abdul Karim, A. Saaban et al., "Construction of cubic timmer triangular patches and its application in scattered data interpolation," Mathematics, vol. 8, no. 2 , p. $159,2020$.

[28] M. A. Evgrafov, "The Abel-Goncharov interplation problem(in Russian)," Gosudastvennoe Izdatel'stvo Tekhnikoteoreticheskio Liteatury, Springer, Moscow, Russian, 1954.

[29] P. H. Davis, Interpolation and Approximation, Blaisdell Publishing Company, New York, NY, USA, 1963.

[30] B. Sendov and A. Andreev, "Approximation and interpolation theory," in Handbook of Numerical Analysis, P. G. Ciarlet and J. L. Lions, Eds., Elsevier Science, 1994.

[31] K. Dzhaparidze and R. H. P. Janssen, "A stochastic approach to an interpolation problem with applications to Hellinger integrals and arithmetic-geometric mean relation-ship," CWI Report, vol. 7, pp. 245-258, 1994.

[32] P. J. Davis, Interpolation and Approximation, Dover Publications Inc, New York, NY, USA, 1975.

[33] R. Caira and F. Dell'Accio, "Shepard--Bernoulli operators," Mathematics of Computation, vol. 76, no. 257, pp. 299-322, 2007. 